\title{
Aktuelle Probleme im Reiserecht durch die Corona-Krise
}

\author{
Privatdozent Dr. Patrick Meier, Würzburg*
}

\section{Inhaltsübersicht}

A. Einleitung 176

B. Kosten für eine ungewollte Verlängerung am Urlaubsort 176

I. Haftung des Reiseveranstalters 177

1. Rechte auf Abhilfe, Rücktritt, Minderung und Schadensersatz 177

2. Ansprüche auf Ersatz von Hotelkosten 179

a) Grundsatz 179

b) Verlängerung 180

II. Haftung des Leistungserbringers 183

III. Ausgleich zwischen Reiseveranstalter und Leistungserbringer 184

C. Stornierungskosten bei Absage durch den Reisenden 186

I. Rücktritt wegen Krankheitsgefahren 186

II. Rücktritt wegen drohender Nachteile nach Rückkehr 188

$\begin{array}{ll}\text { D. Insolvenzsicherung } & 190\end{array}$

I. Geltende Lage im deutschen Recht 190

II. Europarechtliche Vorgaben 191

III. Rechtsfolgen 192

1. Verpflichtung des Absicherers 192

2. Staatshaftung 194

a) Gegenüber den Reisenden 194

b) Gegenüber dem Absicherer 194

3. Vertragsverletzungsverfahren 195

E. Anspruch auf Rückzahlung des Reisepreises $\quad 196$

I. Aktuelle Lage 196

II. Reformüberlegungen des Gesetzgebers 198

1. Rechtspolitische Planungen 198

2. Rechtliche Bewertung 200

3. Konsequenzen einer möglichen Änderung 203

$\begin{array}{ll}\text { F. Schluss } & 204\end{array}$

* Der Autor ist Privatdozent an der Julius-Maximilians-Universität Würzburg und

Notarassessor bei der Landesnotarkammer Bayern. 


\section{A. Einleitung}

Die Corona-Krise trifft die gesamte deutsche, europäische und Weltwirtschaft. Doch nur wenige Bereiche werden härter erschüttert als die Tourismusbranche. Sie leidet mit am stärksten unter den durch die Pandemie ausgelösten Folgen. Die wirtschaftlichen Schäden sind überwiegend katastrophal, nicht selten für die Unternehmen existenzbedrohend. Allein die kommunizierten Verluste der Lufthansa AG übersteigen die Vorstellungskraft der meisten Beobachter. Doch nicht nur die großen Flugkonzerne, sondern auch zahlreiche Reiseanbieter werden in ihrer Existenzgrundlage betroffen. Juristisch spielen sich die zentralen Fragen in den $\mathbb{S} \$ 651$ a ff. BGB und damit im so genannten Reisevertragsrecht sowie in den europäisch geprägten Vorgaben über die Passagierrechte ab. Im Folgenden sollen vier Problemkreise, die sich in der aktuellen Situation als besonders drängend darstellen, aufgegriffen und einer näheren Betrachtung zugeführt werden.

\section{B. Kosten für eine ungewollte Verlängerung am Urlaubsort}

Vor allem zu Beginn der Krise wurde eine Vielzahl von Urlaubern von den ergriffenen staatlichen und behördlichen Maßnahmen noch an ihrem Urlaubsort überrascht. Dies bewirkte in vielen Fällen, dass durch die zumindest vorübergehende Einstellung des Flug- und sonstigen Reiseverkehrs eine Rückreise für diese Personen unmöglich war. Teilweise wurden die Betreffenden sogar unter Quarantäne gestellt und konnten deshalb den Heimflug nicht antreten. So kam es dazu, dass der Aufenthalt am Urlaubsort unfreiwillig verlängert werden musste. Als problematisch in derartigen Situationen erweist sich nicht nur der Umstand, dass am Wohnort Termine nicht wahrgenommen und etwaige arbeitsvertragliche Verpflichtungen nicht erfüllt werden können. Darüber hinaus müssen die Urlauber ihren weiteren, ungeplanten Aufenthalt vor Ort organisieren, was typischerweise bedeutet, die Verträge mit den Hotels oder anderen Dienst- und Werkleistern zu verlängern, um jedenfalls bis zum Rückflug einen Schlafplatz zur Verfügung zu haben. Dies wirft die Frage auf, wer die Kosten für eine derartige erzwungene Ausweitung des Aufenthalts zu tragen hat. 


\section{Haftung des Reiseveranstalters}

\section{Rechte auf Abhilfe, Rücktritt, Minderung und Schadensersatz}

Naheliegend wäre es, den Reiseveranstalter, der für die Reise verantwortlich ist, falls er, wie meist, im Rahmen seines Pflichtenprogramms auch die Beförderung schuldet, auf diese Mehrkosten in Anspruch zu nehmen. Wird allerdings der Reisende selbst unter Quarantäne gestellt, scheidet dies von vornherein aus, weil dann die vertragsgemäße Leistung des Reiseveranstalters nicht betroffen ist. Er bietet seine Leistung vielmehr fehlerfrei an; wenn der Reisende aus persönlichen Gründen sie nicht anzunehmen vermag, begründet dies keine Ansprüche gegen den Veranstalter. ${ }^{1}$ Bestenfalls kann der Reisende in diesem Fall hoffen, dass ihm nach dem öffentlichen Recht des anordnenden Staates ein Entschädigungsanspruch zusteht, der ihm den Ersatz seiner Kosten gestattet. Ebenfalls kein Mangel der Reise ist gegeben, wenn An- und Abreise in der Verantwortlichkeit des Reisenden selbst stehen. Hat sich der Veranstalter zu deren Durchführung nicht verpflichtet, liegen etwaige Erschwerungen oder Ausfälle außerhalb seines Gefahrenbereichs, so dass der Urlauber abseits von $\$ 651 \mathrm{q} \mathrm{BGB}^{2}$ keine Rechte gegen den Reiseveranstalter herleiten kann.

Das Vorliegen eines Reisemangels nach $\$ 651$ i Abs. 1, Abs. 2 S. 1 BGB steht allerdings außer Frage, wenn ein Teil der vereinbarten Reiseleistung nicht zur Durchführung gelangt. ${ }^{3}$ Dies gilt auch für den geschuldeten Rückflug, wenn dieser überhaupt nicht oder jedenfalls nicht zu dem Zeitpunkt, zu dem er vereinbart war, ${ }^{4}$ ausgeführt wird. ${ }^{5}$ In der Konsequenz entstehen für den Reisenden die Ansprüche aus $₫ 651$ i Abs. 3 BGB. Das auf Abhilfe gem. $\$ 651$ i Abs. 3 Nr. 1 i.V.m. $\$ 651$ k Abs. 1 BGB gerichtete Recht nützt ihm aber ebenso wenig wie die Möglichkeit, nach $\$ 651$ i Abs. 3 Nr. 2 i.V.m. $\$ 651$ k Abs. 2 BGB selbst Abhilfe zu schaffen. Kann der Rück-

1 Siehe auch zur Anwendung von $₫ 326$ Abs. 2 S. 1 BGB auf die Fälle des Reiseabbruchs: W. Ernst, in: F. J. Säcker/R. Rixecker/H. Oetker/B. Limperg (Hrsg.), Münchener Kommentar zum BGB, Bd. 3, 8. Aufl. München 2019, \326 Rn. 72; C. Herresthal, in: B. Gsell/W. Krüger/S. Lorenz/C. Reymann (Hrsg.), beckonline.Grosskommentar zum Zivilrecht, 2020, \326 BGB Rn. 249.

2 Siehe zum Anwendungsbereich: C. Sorge, in: B. Gsell/W. Krüger/S. Lorenz/C. Reymann (Hrsg.), beckonline.Grosskommentar zum Zivilrecht, 2020, $\$ 651 \mathrm{q} \mathrm{BGB,}$ Rn. 45 ff.

3 BGHZ 97, 255 (258 ff.).

4 Vgl. auch BGH NJW 2014, 1168 (1169); NJW 2014, 3721 (3721 f.).

5 Siehe zur Kasuistik: E. Führich, in E. Führich/A. Staudinger (Hrsg.), Reiserecht, 8. Aufl. München 2019, Anh. zu \21 Rn. 16 ff. 
flug mangels Angebots am Markt insgesamt nicht realisiert werden, ist die Erfüllung der Ansprüche i. S. d. $\$ 275$ Abs. 1 BGB unmöglich; die darauf gerichteten Befugnisse bleiben daher zumindest praktisch ohne Wirkung. Ein Anspruch auf Abhilfe durch andere Reiseleistungen nach $\$ 651$ i Abs. 3 Nr. 3 i.V.m. $\$ 651$ k Abs. 3 BGB muss gleichermaßen ausscheiden, weil dieser sich nur auf Reiseleistungen richten kann, die in etwa gleichwertig zur nicht erbringbaren Reiseleistung sind. ${ }^{6}$ Dies ist allerdings bei weiteren Übernachtungen im Vergleich mit der Rückbeförderung nicht der Fall, weil es bei der Beförderung um eine Veränderung des Aufenthaltsortes geht; der Verbleib am Ort entspricht dem noch nicht einmal ansatzweise. Offensichtlich gleichermaßen nicht hilfreich sind die Befugnisse zur Kündigung und Minderung gem. $\$ 651$ i Abs. 3 Nr. 5 i.V.m. $\$ 6511$ BGB bzw. $\$ 651$ i Abs. 3 Nr. 6 i.V.m. $\$ 651 \mathrm{~m}$ BGB. In der Sache nützlich wäre demgegenüber zwar ein Schadensersatzanspruch aus $\$ 651$ i Abs. 3 Nr.7 i.V.m. $\$ 651 \mathrm{n} \mathrm{BGB}$, mit dem sämtliche Mehrkosten sowohl am Reiseort als auch alle Folgeschäden, die durch die verspätete Rückreise entstehen, beim Reiseveranstalter liquidiert werden können. ${ }^{7}$ Allerdings ist ein solches Recht wegen $\$ 651 \mathrm{n}$ Abs. 1 Nr. 3 BGB ausgeschlossen, wenn der Reisemangel durch unvermeidbare, außergewöhnliche Umstände ${ }^{8}$ verursacht wurde. Nach Erwägungsgrund 31 der Pauschalreise-RL ${ }^{9}$ zählen hierzu unter anderem erhebliche Risiken für die menschliche Gesundheit, wie insbesondere schwere Krankheiten am Reiseziel. Ohne bereits jetzt schon abschließend die medizinischen Auswirkungen der Covid 19-Pandemie beurteilen zu können, lässt sich diese aber sicher unter die von der Richtlinie aufgestellten Begrifflichkeiten fassen. ${ }^{10}$ Es dürfte zudem nicht darauf ankommen, ob

6 G. Deppenkemper, in H. Prütting/G. Wegen/G. Weinreich (Hrsg.), 14. Aufl. Köln 2019, $\sqrt{ } 651$ k Rn. 9; S. A. Geib, in: H. G. Bamberger/H. Roth/W. Hau/R. Poseck (Hrsg.), Beck'scher Online-Kommentar BGB, \$ 651 k Rn. 21.

7 BT-Drs. 18/10822, 83.

8 Siehe zum Begriff: S. Bergmann/D. Blankenburg, Unvermeidbare außergewöhnliche Umstände im Pauschalreise- und Luftverkehrsrecht, NJW 2019, 3678 (3680 ff.); J. D. Harke, in: B. Gsell/W. Krüger/S. Lorenz/C. Reymann (Hrsg.), beckonline.Grosskommentar zum Zivilrecht, 2020, $\$ 651$ h Rn. 42 f.; B. Steinrötter, in: M. Herberger/M. Martinek/H. Rüßmann/S. Weth/M. Würdinger (Hrsg.), jurisPraxiskommentar zum BGB, Bd. 2, 9. Aufl. Saarbrücken 2020, $\$ 651$ h Rn. $21 \mathrm{ff}$.

9 Richtlinie (EU) 2015/2302 des Europäischen Parlaments und des Rates vom 25. November 2015 über Pauschalreisen und verbundene Reiseleistungen, zur Änderung der Verordnung (EG) Nr. 2006/2004 und der Richtlinie 2011/83/EU des Europäischen Parlaments und des Rates sowie zur Aufhebung der Richtlinie 90/314/EWG des Rates, ABl. L 326, S. 1 ff.

10 Ebenso: A. Staudinger/C. Achilles-Pujol, in: H. Schmidt, Covid 19, Rechtsfragen zur Corona-Krise, 2020, $\$ 7$ Rn. 51. 
am Reiseziel selbst die Krankheit ausbricht ${ }^{11}$ oder die Störung der Rückreise darauf beruht, dass im Heimatland des Reisenden eine derartige Krankheit herrscht, und daher infolge behördlicher Anordnungen der Flug- und Reisebetrieb unterbunden ist. Auch in letzterer Konstellation gebieten die Wertungen der Richtlinie eine Exkulpation des Reiseveranstalters und damit die Verneinung eines Schadensersatzanspruchs, weil ihn für die Handlungen der lokalen Behörden keine Verantwortlichkeit trifft und er deshalb für diese auch nicht einstehen muss. Eine Ausnahme wäre nur dann zu machen, wenn der Reiseveranstalter die Schwierigkeiten vorhersehen hätte können und keine Maßnahmen zur Abwendung getroffen hat. ${ }^{12}$

\section{Ansprüche auf Ersatz von Hotelkosten}

\section{a) Grundsatz}

Gänzlich schutzlos ist der Reisende gleichwohl nicht. Ihm verbleibt in derartigen Situationen das eher versteckte und meist nicht so bedeutsame Recht aus $₫ 651$ i Abs. 3 Nr. 4 i.V.m. $\$ 651$ k Abs. 4 und Abs. 5 BGB. Gemäß $\$ 651$ k Abs. 4 BGB kann der Reisende in dem Fall, dass ihm die Rückbeförderung geschuldet ist und diese auf Grund unvermeidbarer, außergewöhnlicher Umstände unterbleibt, ${ }^{13}$ vom Reiseveranstalter verlangen, dass er die Kosten für eine notwendige Beherbergung des Reisenden für die Dauer von höchstens drei Nächten ${ }^{14}$ übernimmt. Auf diese Weise kann der Reisende jedenfalls einen Teil seiner Ausgaben beim Reiseveranstalter liquidieren. Das Recht ist grundsätzlich von einer Mangelanzeige nach $\$ 651$ o Abs. 1 BGB unabhängig, wie $\$ 651$ o Abs. 2 BGB zeigt, der nur die Rechte aus $₫ 651 \mathrm{~m}$ und $\$ 651 \mathrm{n}$ BGB ausschließt. ${ }^{15}$ Zudem wäre hier eine Anzeige entbehrlich, da der Reiseveranstalter den Mangel kennt und die

11 Siehe dazu auch: AG Düsseldorf RRa 2014, 56; RRa 2016, 25; AG Frankfurt a.M. RRa 2011, 144; AG Rüsselsheim RRa 2016, 88 (89) (zu Erkrankungen von Passagieren).

12 Staudinger/Achilles-Pujol (Fn. 10), \$ 7 Rn. 51.

13 Handelt es sich nicht um solche, steht dem Reisenden nach $\$ 651$ n BGB ein Schadensersatzanspruch zu.

14 Krit. hierzu: Sorge (Fn. 2), \$ 651 k Rn. 153.

15 Siehe zur Reichweite des Ausschlusses: Geib (Fn. 6), \$ 651 o Rn. 14. 
Obliegenheit richtigerweise ${ }^{16}$ nicht dazu dient, den Reiseveranstalter über Umstände in Kenntnis zu setzen, die ihm bereits bekannt sind. ${ }^{17}$

\section{b) Verlängerung}

Dem Reisenden steht darüber hinaus nach $\$ 651 \mathrm{k}$ Abs. 5 Nr. 2 BGB unter den dort genannten Bedingungen sogar der Kostenersatz für die volle Dauer der Verzögerung zu. Dies gilt zunächst, wenn es sich um Personen mit eingeschränkter Mobilität nach Art. 2 lit. a VO 1107/2006, ${ }^{18}$ um Schwangere, um unbegleitete Minderjährige oder um Personen, die besonderer medizinischer Betreuung bedürfen, handelt. Voraussetzung ist allerdings, dass dieser Umstand dem Reiseveranstalter mindestens 48 Stunden vor Beginn der Reise angezeigt ${ }^{19}$ wurde. Infolgedessen ist ein Reisender, der erst im Laufe der Reise erkrankt und deshalb medizinische Betreuung benötigt, von der Privilegierung nicht umfasst; insbesondere eine Corona-Infektion am Reiseort bietet daher keinen Anknüpfungspunkt für Zahlungen über die Drei-Tages-Grenze hinaus.

Als für den Reisenden mitunter deutlich wichtiger erweist sich deshalb der in $\ 651 \mathrm{k}$ Abs. 5 Nr. 1 BGB geregelte Ausschluss der Begrenzungsmöglichkeit. Hiernach darf der Reiseveranstalter seine Leistungen nicht auf die Dauer von drei Tagen begrenzen, wenn ein Leistungserbringer für die Beförderung dem Reisenden entsprechende Unterstützungsmaßnahmen für einen längeren Zeitraum nach den Vorgaben der Europäischen Union schuldet. Bedeutsam ist dabei zunächst, dass es nicht auf die Bindung des Reiseveranstalters selbst ankommt, sondern auf die des durch ihn beauf-

16 Wie hier: Geib (Fn. 6), $\$ 651$ o Rn. 3; A. Staudinger, in E. Führich/A. Staudinger (Hrsg.), Reiserecht, 8. Aufl. München 2019, $\mathbb{1} 18$, Rn. 2; A. Staudinger/C. Aslan, Pauschalreiserecht de lege lata und ferenda: Wie weit reicht die Obliegenheit des Reisenden, Mängel gegenüber dem Veranstalter anzuzeigen?, JR 2018, 217 (217 ff.); K. Tonner, in: in: F. J. Säcker/R. Rixecker/H. Oetker/B. Limperg (Hrsg.), Münchener Kommentar zum BGB, Bd. 6, 8. Aufl. München 2020, 651 o, Rn. 8.

17 Anders aber: BGH, NJW 2016, 3304 (3305); LG Duisburg, NJOZ 2006, 2278 (2279); S. Bergmann, in: M. Tamm/K. Tonner/T. Brönneke (Hrsg.), Verbraucherrecht, 3. Aufl. Baden-Baden 2020, $\mathbb{1 8}$ a Rn. 114; D. Paulus, Das neue Pauschalreisevertragsrecht, JuS 2018, 647 (652).

18 Verordnung (EG) Nr. 1107/2006 des Europäische Parlaments und des Rates vom 5. Juli 2006 über die Rechte von behinderten Flugreisenden und Flugreisenden mit eingeschränkter Mobilität, ABl. L 204, S. $1 \mathrm{ff}$.

19 Für niedrige Anforderung: Geib (Fn. 6), \$651 k Rn. 30; strengere Maßstäbe aber empfehlend: Steinrötter (Fn. 8), \$651 k Rn. 39. 
tragten Leistungserbringers, ${ }^{20}$ so dass der Reiseveranstalter nach diesen Regeln auch dann verpflichtet ist, wenn er selbst entsprechende Unterstützungsleistungen nicht anbieten muss. Praktisch bedeutsam erweist sich dies vor allem im Falle von Flugreisen. Nach Art. 5 Abs. 1 lit. b i.V.m. Art. 9 Abs. 1 lit. b der Fluggastrechteverordnung ${ }^{21}$ muss der Beförderer im Falle der Annullierung des Fluges für unbegrenzte Zeit die Hotelkosten tragen. ${ }^{22}$ Er wird weder durch ein mangelndes Verschulden noch durch sonstige Billigkeitsaspekte von dieser Verpflichtung frei. ${ }^{23}$ Eine analoge Anwendung des Art. 5 Abs. 3 der Fluggastrechteverordnung, der bei außergewöhnlichen Umständen zumindest den Entschädigungsbetrag entfallen lässt, hat der EuGH verneint. ${ }^{24}$ Ist damit eine Flugreise geschuldet, die von der Fluggastrechteverordnung erfasst wird, ${ }^{25}$ muss der Reiseveranstalter in unbegrenztem Umfang die anfallenden Hotelkosten nach $\$ 651 \mathrm{k}$ Abs. 4 , Abs. 5 BGB übernehmen.

Nicht in gleicher Weise geschützt ist allerdings der Reisende, der mit einem anderen Verkehrsmittel den Weg zwischen Wohn- und Reiseort zurücklegt. Hat er eine Bahnreise gebucht, greift Art. 18 VO 1371/200726 nicht, der allein für Verspätungen gilt. ${ }^{27}$ Vielmehr ist bei einer Annullierung ausschließlich Art. 15 VO 1371/2007 anzuwenden, der auf Art. 32 $\mathrm{CIV}^{28}$ verweist. Die Norm sieht zwar in ihrem Abs. 1 S. 2 einen Schadens-

20 Geib (Fn. 6), \$651 k Rn. 30; A. Staudinger, in: R. Schulze (Hrsg.), BGB, 10. Aufl. Baden-Baden 2019, $\$ 651$ k Rn. 5; Tonner (Fn. 16), $\$ 651$ k Rn. 38;

21 Verordnung (EG) Nr. 261/2004 des Europäischen Parlaments und des Rates vom 11. Februar 2004 über eine gemeinsame Regelung für Ausgleichs- und Unterstützungsleistungen für Fluggäste im Fall der Nichtbeförderung und bei Annullierung oder großer Verspätung von Flügen und zur Aufhebung der Verordnung (EWG) Nr. 295/91, ABl. L 46, 1 ff.

22 B. Steinrötter, in: B. Gsell/W. Krüger/S. Lorenz/C. Reymann (Hrsg.), beckonline.Grosskommentar zum Zivilrecht, 2020, Art. 9 Fluggastrechte-VO Rn. 28.

23 Bergmann (Fn. 17), $\mathbb{1} 18$ a Rn. 127; H. Hopperdietzel, in: R. Schmid (Hrsg.), Beck'scher Online Kommentar zur Fluggastrechte-Verordnung, 14. Ed. 2020; rechtspolitische Änderungsvorschläge unterbreitend: Steinrötter (Fn. 22), Art. 9 Fluggastrechte-VO Rn. 52.

24 EuGH Slg. 2011, I-3985 Rn. 23; NJW 2013, 921 (923).

25 Vgl. zum Anwendungsbereich Art. 3 der Fluggastrechteverordnung.

26 Verordnung (EG) Nr. 1371/2007 des Europäischen Parlaments und des Rates vom 23. Oktober 2007 über die Rechte und Pflichten der Fahrgäste im Eisenbahnverkehr, ABl. L 315, $14 \mathrm{ff}$.

27 Ebenso: H. Lindemann, Neue Fahrgastrechte im Eisenbahnverkehr, TranspR 2011, 10 (13); P. Meier, in: M. Tamm/K. Tonner/T. Brönneke (Hrsg.), Verbraucherrecht, 3. Aufl. Baden-Baden 2020, $\mathbb{1} 18$ b Rn. 44.

28 Übereinkommen über den internationalen Eisenbahnverkehr in der Fassung des Protokolls von Vilnius in Kraft ab 1.7.2006. 
ersatzanspruch vor, allerdings ist dieser nach Art. 32 Abs. 2 lit. a CIV bei außergewöhnlichen Umständen, zu denen die Corona-Krise ebenfalls zu zählen ist, ausgeschlossen. Eine weitergehende Unterstützungsverpflichtung existiert für Bahnreisen im Falle der Annullierung nicht. Insbesondere kann der auf Verspätungen bezogene Art. 18 VO 1371/2007 nicht analog auf die Annullierung angewendet werden, weil der Gesetzgeber durch die Fassung zu erkennen gibt, dass er das Problem gesehen hat, dessen Regelung aber allein dem CIV überlassen wollte. ${ }^{29}$ Auch für Busreisen ergeben sich keine weitergehenden Rechte der Passagiere, die den Reiseveranstalter nach $₫ 651$ k Abs. 5 Nr. 1 BGB zu einer umfassenderen Übernahme der Hotelkosten zwingen würden. Gem. Art. 21 lit. b VO 181/201130 schuldet zwar der Beförderer die Übernahme von Hotelkosten bei einer Annullierung, allerdings darf er die Kostenübernahme auf maximal zwei Nächte beschränken, so dass die Vorgaben des $₫ 651$ k Abs. 4 BGB sogar weitergehend sind. Bei einer Reise mit einem Binnenschiff ${ }^{31}$ bleibt ebenfalls kein Raum für $₫ 651$ k Abs. 5 Nr. 1 BGB. Nach $₫ 17$ Abs. 2 VO 1177/2010 32 ist der Beförderer bei einer Annullierung zur Übernahme von Hotelkosten an Land nur für die Dauer von drei Nächten und damit in gleichem Umfang, wie von $\$ 651$ k Abs. 4 BGB vorgesehen, verpflichtet. Dem Reisenden ist allerdings insoweit zumindest ein wenig geholfen, weil die Übernachtung an Bord nach dieser Vorschrift kostenlos zu erfolgen hat, so dass der Reiseveranstalter wenigstens hierfür keine weiteren Zahlungen verlangen kann.

Hat der Reisende Kosten für angefallene weitere Übernachtungen bereits gegenüber dem Hotel beglichen, so kann er unmittelbar aus $\$ 651 \mathrm{k}$ Abs. 4 BGB die Erstattung vom Reiseveranstalter verlangen. ${ }^{33}$ Die Fassung der Norm ist ausreichend offen, um einen unmittelbaren Zahlungsan-

29 Siehe zur kompetenziellen Möglichkeit der abweichenden Regelung: R. Freise, in: R. Herber/C. Schmidt (Hrsg.), Münchener Kommentar zum Handelsgesetzbuch, Bd. 7, 4. Aufl. München 2020, COTIF, Einleitung Rn. 42 f.

30 Verordnung (EU) Nr. 181/2011 des Europäischen Parlaments und des Rates vom 16. Februar 2011 über die Fahrgastrechte im Kraftomnibusverkehr und zur Änderung der Verordnung (EG) Nr. 2006/2004, ABl. L 55, 1 ff.

31 Vgl. dazu Art. 2 VO 1177/2010.

32 Verordnung (EU) Nr. 1177/2010 des Europäischen Parlaments und des Rates vom 24. November 2010 über die Fahrgastrechte im See-und Binnenschiffsverkehr und zur Änderung der Verordnung (EG) Nr. 2006/2004, ABl. L 334, 1 ff.

33 H. Brox/W. D. Walker, Besonderes Schuldrecht, 43. Aufl. München 2019, $\mathbb{} 28$ Rn. 37; C. Förster, Das neue Pauschalreiserecht in Studium und Examen, JA 2018, 561 (567); Geib (Fn. 6), \651 k Rn. 29; Paulus, Pauschalreisevertragsrecht (Fn. 17), JuS 2018, 647 (653); Steinrötter (Fn. 8), \$651 k Rn. 52; für ein Verpflichtung zur Unterbringung dagegen: Staudinger (Fn. 20), \$651 k Rn. 5; 
spruch zu tragen. Das Forderungsrecht beschränkt sich nicht darauf, dass der Reiseveranstalter eine Unterkunft selbst zur Verfügung stellen, sondern sie ist so ausgestaltet, dass er etwa anfallende Kosten übernehmen muss. Dies rechtfertigt es, aus der Norm selbst einen Anspruch auf Leistung des erforderlichen Geldbetrages abzuleiten. ${ }^{34}$ Weil die für die Wartezeit gewählte Unterkunft zu der bisherigen gleichwertig sein soll, ist es grundsätzlich nicht zu beanstanden, wenn der Reisende in seinem aktuellen Hotel verbleibt. Eine bessere Vergleichbarkeit als die Fortsetzung der bestehenden Unterbringung dürfte sich kaum erreichen lassen, so dass ein solches Vorgehen dem Geist der Regelung vollauf entspricht.

\section{Haftung des Leistungserbringers}

Infolge der dargestellten Regelungen kann der Kunde sich zudem auch jeweils unmittelbar an den Leistungserbringer halten, soweit dieser nach den oben beschriebenen Grundsätzen ${ }^{35}$ gemäß den europäischen Verordnungen zur Übernahme der Hotelkosten verpflichtet ist. ${ }^{36}$ Dies erweist sich insbesondere für die Touristen als nützlich, die keine Pauschalreise ${ }^{37}$ nach den $\$ \$ 651$ a ff. BGB gebucht haben oder deren Reise die Beförderung nicht umfasst. In diesem Fall scheidet der Anspruch gegen einen Reiseveranstalter gem. $\$ 651 \mathrm{k}$ Abs. 4 BGB aus, so dass nur Rechte gegen den Leistungserbringer verbleiben. Auch befinden sich die Fluggesellschaften in einer deutlich schlechteren Position als die sonstigen Beförderungsunternehmen. Die Bahn bleibt von derartigen Ansprüchen vollkommen frei, Anbieter von Busreisen und von Binnenschifffahrten können ihre Kosten zumindest beschränken. ${ }^{38}$

34 Sorge (Fn. 2), $\$ 651 \mathrm{k} \mathrm{Rn.} 158$.

35 Oben B. I. 2. b).

36 So wohl auch Steinrötter (Fn. 8), \$651 k Rn. 38, der davon ausgeht, die Verpflichtungen der Leistungserbringer blieben „unberührt“.

37 Dazu D. Looschelders, Schuldrecht Besonderer Teil, 15. Aufl. München 2020, $\$ 36$ Rn. 3 ff.; D. Medicus/S. Lorenz, Schuldrecht II Besonderer Teil, 18. Aufl. München

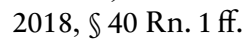

38 Oben B. I. 2. b). 


\section{Ausgleich zwischen Reiseveranstalter und Leistungserbringer}

Sind Reiseveranstalter und Leistungserbringer nicht identisch, stellt sich die Frage, wie die entstandenen Kosten zwischen beiden zu verteilen sind. Relevanz entfaltet dies nur insoweit, als in den Verträgen zwischen Veranstalter und Leistungserbringer keine hierauf bezogenen Bestimmungen aufgenommen sind. Existieren solche, gehen sie den gesetzlichen Vorgaben vor und verdrängen diese..$^{39}$ Andernfalls kann es zu einem gesetzlichen Regress nur kommen, soweit Reiseveranstalter und Beförderungsdienstleister Gesamtschuldner gem. $\$ 421 \mathrm{BGB}$ sind. ${ }^{40}$ Dies ist der Fall, soweit der Reiseveranstalter nach $\$ 651$ k Abs. 4, Abs. 5 BGB und der Leistungserbringer nach den für ihn einschlägigen Vorgaben gemäß der europäischen Verordnungen verpflichtet sind. Sieht sich der Leistungserbringer einer Verpflichtung aus der Verordnung und der Reiseveranstalter einer solchen aus $₫ 651 \mathrm{k}$ Abs. $4 \mathrm{BGB}^{41}$ ausgesetzt, dürfte unabhängig davon, wer in Anspruch genommen wird, an der grundsätzlichen Regel des $\$ 426$ Abs. 1 BGB festzuhalten sein, weshalb die Kosten nach Köpfen verteilt werden. Dies ergibt sich daraus, dass beide Schuldner gegenüber dem Reisenden einer eigenen, selbstständigen Verpflichtung unterliegen, so dass es angemessen ist, sie auch im Innenverhältnis gleich zu behandeln. Der in Anspruch Genommene kann mithin die Hälfte der aufgewendeten Ausgaben vom jeweils anderen erstattet verlangen. Reichen die Rechte gegen den Reiseveranstalter weiter als die gegen den Leistungserbringer, wie dies vor allem bei Bahn- und Busreisen der Fall ist, ${ }^{42}$ kommt für die überschießende Verpflichtung des Reiseveranstalters eine Ausgleichsmöglichkeit ohnehin nicht in Betracht, weil es insoweit bereits an der Gesamtschuldnerschaft fehlt.

Rechtlich schwieriger zu beurteilen ist dagegen die nur für die Luftbeförderung denkbare Situation, wonach auf Grund der Verpflichtung des Beförderers auch die des Reiseveranstalters wegen $\$ 651$ k Abs. 5 Nr. 1 BGB ausgedehnt wird. In einer solchen Konstellation dürfte die zumindest hälf-

39 S. Heinemeyer, in: F. J. Säcker/R. Rixecker/H. Oetker/B. Limperg (Hrsg.), Münchener Kommentar zum BGB, Bd. 3, 8. Aufl. München 2019, $₫ 426$ Rn. 16; D. Looschelders, in: M. Löwisch (Red.), J. von Staudingers Kommentar zum Bürgerlichen Gesetzbuch mit Einführungsgesetz und Nebengesetzen, Berlin 2017, $\$ 426$ Rn. 5.

40 Siehe zu den Voraussetzungen J. D. Harke, Allgemeines Schuldrecht, Berlin, Heidelberg 2010, Rn. $450 \mathrm{ff}$.

41 Also für die ersten drei Tage der Verzögerung, oben B. I. 2. a).

42 Oben B. I. 2. b). 
tige Übernahmeverpflichtung des Leistungserbringers aus $\$ 426$ Abs. 1 BGB nicht in Zweifel zu ziehen sein, weil der Reiseveranstalter nicht schlechter stehen darf als im Anwendungsbereich des $\$ 651 \mathrm{k}$ Abs. 4 BGB. Blickt man allerdings auf die erkennbare Intention des $\$ 651 \mathrm{k}$ Abs. $5 \mathrm{Nr} .1$ $\mathrm{BGB}$, folgt hieraus sogar eine andere Bestimmung im Sinne des $\$ 426$ Abs. 1 BGB $^{43}$ mit der Konsequenz, dass der Leistungserbringer die über die drei Übernachtungen hinausgehenden Mehrkosten im Innenverhältnis allein zu tragen hat. $\$ 651$ k Abs. 5 Nr. 1 BGB knüptt ebenso, wie die ihm zu Grunde liegende Vorgabe in Art. 13 Abs. 8 Pauschalreise-RL nur an die Verpflichtungen an, denen der Beförderer unterliegt, und erklärt diese als alleinigen Grund für die Ausweitung der Kostentragungspflicht des Reiseveranstalters. Offenbar soll damit dem Reisenden eine Verbesserung dadurch gewährt werden, dass er sich nicht mit zwei unterschiedlichen Schuldnern auseinandersetzen muss, sondern seine Ansprüche gebündelt gegen den Reiseveranstalter durchsetzen kann. ${ }^{44}$ Dass damit aber zugleich eine auch nur teilweise Befreiung des Beförderers von den Kosten im Innenverhältnis gewollt war, lässt sich nicht erkennen, zumal Art. 22 Pauschalreise-RL gerade die Möglichkeiten zum Regress eröffnet und damit klarstellt, dass das Verhältnis nach außen nicht auch das nach innen beherrscht. Es ist vielmehr anzunehmen, dass sich die Privilegierung exklusiv auf den Reisenden erstrecken sollte. Betrachtet man diese Wertungen, zeigen sie, dass der Luftbeförderer, soweit seine Verpflichtung gem. $\$ 651 \mathrm{k}$ Abs. 5 Nr. 1 BGB auch eine Vermehrung der Ansprüche des Reisenden gegen den Reiseveranstalter bewirkt, diesen im Innenverhältnis vom Mehraufwand gänzlich freizustellen hat. Weil alleinige Bedingung für die Verpflichtung des Reiseveranstalters die des Beförderers ist, fordert die Wertung des Art. 22 Pauschalreise-RL die Auferlegung der finanziellen Nachteile im Innenverhältnis an den Beförderer.

43 Siehe zur Frage ungleichstufiger Gesamtschuldner, bei denen einer der Beteiligten nur für das Verschulden des anderen im Außenverhältnis einstehen muss: $M$. Gehrlein, in: H. G. Bamberger/H. Roth/W. Hau/R. Poseck (Hrsg.), Beck'scher Online-Kommentar BGB, $\ 426$ Rn. 11; Heinemeyer (Fn. 39), $\ 426$ Rn. 22; B. Kreße, in: B. Gsell/W. Krüger/S. Lorenz/C. Reymann (Hrsg.), beckonline.Grosskommentar zum Zivilrecht, 2020, $₫ 426$ BGB Rn. 73.

44 Als „Harmonisierung“ bezeichnet Staudinger (Fn. 20), $\$ 651$ k Rn. 5 dies. Von einem „Durchschlagen“ spricht die Gesetzbegründung in BT-Drs. 18/10822, 81. 


\section{Stornierungskosten bei Absage durch den Reisenden}

Von Relevanz sind des Weiteren mögliche Stornierungskosten, wenn der Reisende vom Vertrag zurücktritt, weil er im Zuge der Pandemie eine Reise nicht durchführen will. Teilweise äußern Kunden im Zusammenhang mit der Krise das Gefühl, dass die Reiseveranstalter mit Absagen ihrerseits möglichst lange zögern, um etwa vereinbarte Stornierungsgebühren verlangen zu können, wenn die Reisenden den Rücktritt erklären. Unabhängig davon, ob diese Eindrücke die Wahrheit widerspiegeln, könnte auch ein derartiges Vorgehen rechtlich die Verpflichtung zur vollen Rückzahlung des Reisepreises aber nicht vermeiden.

\section{Rücktritt wegen Krankheitsgefahren}

Nach $₫ 651$ h Abs. 1 S. 1 BGB darf der Reisende jederzeit vor Beginn der Reise $^{45}$ vom Vertrag zurücktreten. In diesem Fall entfällt nach $\$ 651 \mathrm{~h}$ Abs. 1 S. 2 BGB der Anspruch auf den vereinbarten Reisepreis, allerdings steht nach Abs. 1 S. 3 der Vorschrift dem Reiseveranstalter eine angemessene Entschädigung zu. Diese ist entweder gem. $\$ 651$ h Abs. 2 S. 2 BGB konkret zu berechnen ${ }^{46}$ oder kann durch angemessene Entschädigungspauschalen nach $\$ 651$ h Abs. 2 S. 1 BGB bereits im Vorfeld bestimmt werden. ${ }^{47}$ Legt man dies zu Grunde, stünde dem Reiseveranstalter in der Tat die Möglichkeit offen, auf ein frühzeitiges Handeln des Reisenden zu spekulieren und so zumindest einen Teil des Reisepreises behalten zu können. Es wäre dann zu erwägen, ob das Ergebnis nicht auf Grund eines Schadensersatzanspruch $s^{48}$ oder im Rahmen des $₫ 242$ BGB zu korrigieren wäre.

Für derartige Kunstgriffe besteht aber kein Anlass, weil der Gesetzgeber diese Situation in $\$ 651 \mathrm{~h}$ Abs. 3 BGB einer Lösung zugeführt hat. Hiernach kann nämlich durch den Reiseveranstalter keine Entschädigung verlangt werden, wenn am Reiseort oder in dessen unmittelbarer Nähe unvermeidbare, außergewöhnliche Umstände auftreten, die die Durchführung der Pauschalreise oder die Beförderung an den Reiseort erheblich beein-

45 Siehe zum Begriff Harke (Fn. 8), $\$ 651$ h Rn. 15.

46 Hierzu: Tonner (Fn. 16), $\$ 651$ h Rn. $23 \mathrm{ff}$.

47 Dazu: Staudinger (Fn. 16), $\$ 16$ Rn. $12 \mathrm{ff}$.

48 Ein solcher wäre unter Annahme einer Schutzpflichtverletzung denkbar, da der Reiseveranstalter schnellstmöglich bekanntgeben muss, dass er seine Leistung nicht erbringen kann, um den Reisenden vor weiteren Nachteilen zu bewahren. 
trächtigen. Mit der Fassung der Vorschrift, die sich an die Regelungen des ihr zu Grunde liegenden Art. 12 Abs. 2 Pauschalreise-RL anlehnt, nimmt der Gesetzgeber erkennbar Bezug auf Art. 5 Abs. 3 Fluggastrechteverordnung. ${ }^{49}$ Insoweit sind nun parallele Strukturen geschaffen, ${ }^{50}$ die die frühere Regelung für den Rücktritt vor Reisebeginn, die noch die „höhere Ge-

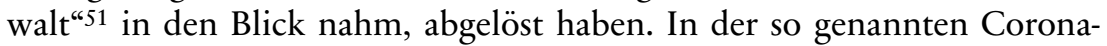
Pandemie bleiben die Änderungen aber ohne Belang. Diese ist in jedem Fall ein unvermeidbares und außergewöhnliches Ereignis im Sinne des $\$ 651$ h Abs. 3 BGB. ${ }^{52}$ Erwägungsgrund 31 der Pauschalreise-RL nennt explizit erhebliche Risiken für die menschliche Gesundheit, vor allem Ausbrüche schwerer Krankheiten. Zeigen sich damit gehäufte Corona-Erkrankungen am Reiseort oder in dessen Nähe, ${ }^{53}$ ergibt sich bereits aus der Wertung des Erwägungsgrundes 31, dass der Reisende zum Rücktritt ohne Entschädigung berechtigt ist. ${ }^{54}$ Doch selbst wenn am Reiseort kein erhöhtes Risiko besteht, werden typischerweise unvermeidbare und außergewöhnliche Umstände vorliegen. Existieren behördliche Maßnahmen, ${ }^{55}$ nach denen entweder die Einreise in das Zielland unmöglich oder mit einer Quarantäne verbunden ist ${ }^{56}$ oder wesentliche Aktivitäten vor Ort nicht erfolgen können, ist gleichermaßen die Durchführung der Reise erheblich beeinträchtigt. Dies gilt vor allem, falls die Hotels nicht geöffnet

49 Bergmann/Blankenburg, Umstände (Fn. 8), NJW 2019, 3678 (3680); Geib (Fn. 6), $\$ 651$ h Rn. 17; H. Sprau, in O. Palandt (Begr.), Bürgerliches Gesetzbuch, 79. Aufl. 2019, $\$ 651$ h Rn. 11; Steinrötter (Fn. 8), $\$ 651$ h Rn. 17.

50 So auch BT-Drs. 18/10822, 76.

51 Dazu ausführlich A. Staudinger, in: D. Kaiser (Red.), J. von Staudingers Kommentar zum Bürgerlichen Gesetzbuch mit Einführungsgesetz und Nebengesetzen, Berlin 2016, \615 e Rn. 15 ff.

52 Epidemien waren auch schon als Fälle der höheren Gewalt nach $₫ 651 \mathrm{j}$ BGB a. F. anerkannt: E. Führich, Umwelteinflüsse bei Pauschalreisen und ihre Konfliktlösungen im Reisevertragsrecht, NJW 1991, 2192 (2194); A. Teichmann, in: R. Stürner (Hrsg.), Jauernig Bürgerliches Gesetzbuch, 17. Aufl. München 2018, 651 j Rn. 2; K. Tonner, Auswirkungen von Krieg, Epidemie und Naturkatastrophe auf den Reisevertrag, NJW 2003, 2783.

53 Zum Begriff: S. Löw, Pauschalreiserecht in Zeiten der Covid-19-Pandemie, NJW 2020, 1252 (1253).

54 Ebenso: Löw, Pauschalreiserecht (Fn. 53), NJW 2020, 1252 (1253); Staudinger/ Achilles-Pujol (Fn. 10), $\$ 7$ Rn. 27.

55 Auch OLG Frankfurt, NJW-RR 2005, 282 (283) erkennt grundsätzlich behördliche Maßnahmen an. Im konkreten Einzelfall (Ungültigkeit der Reisepässe) unter Bezugnahme auf die höhere Gewalt aber anders: BGH, NJW 2017, 2677 (2678f.).

56 Anders aber offenbar Staudinger/Achilles-Pujol (Fn. 10), \$7 Rn. 27. 
haben, ${ }^{57}$ aber auch dann, wenn keine ausreichende Bewegungsfreiheit besteht ${ }^{58}$ oder lokale Sehenswürdigkeiten nicht besucht werden können. In all diesen Fällen kann die Reise nicht wie geplant verwirklicht werden, so dass ein Fall des $₫ 651$ h Abs. 3 BGB gegeben ist. Auch eine Reisewarnung der Bundesregierung oder des Auswärtigen Amtes führt regelmäßig zu einem kostenfreien Rücktrittsrecht vor Reisebeginn. ${ }^{59}$

\section{Rücktritt wegen drohender Nachteile nach Rückkehr}

Als problematisch erweist sich allein die Situation, in der die Reise am Reiseort in keiner Weise beeinträchtigt ist, allerdings nach der Rückkehr mit einer Quarantäne im Heimatstaat gerechnet werden muss. Aktuell zeichnen sich derartige Konstellationen zwar nicht ab, es scheint aber denkbar, dass in Zukunft in bestimmten Staaten oder Teilen davon keine weiteren Maßnahmen gegen das Virus ergriffen werden und damit während der Reise keine Einschränkungen bestehen, jedoch nach Wiedereinreise zum Schutz der lokalen Bevölkerung im Heimatstaat Restriktionen gelten oder sogar eine Verpflichtung zur Quarantäne ausgesprochen wird. Betrachtet man den Wortlaut des $₫ 651$ h Abs. 3 BGB und des Art. 12 Abs. 2 Pauschalreise-RL, muss dieser Aspekt jedoch ohne Bedeutung bleiben. Es kommt nämlich allein darauf an, ob die Umstände, die am Bestimmungsort oder dessen unmittelbarer Nähe herrschen, die Durchführung der Pauschalreise oder die Beförderung der Reisenden an den Reiseort beeinträchtigen. Beides ist bei Maßnahmen, die erst nach der Rückkehr der Reisenden gegen sie verhängt werden, nicht gegeben. Auch eine analoge Anwendung der Vorschrift muss ausscheiden. Infolge der klaren Fassung der Norm, die allein auf den Hintransport abstellt, scheint bereits eine planwidrige Regelungslücke ${ }^{60}$ unwahrscheinlich, da der Gesetzgeber sich offenbar darüber bewusst war, dass er die Rückbeförderung so nicht erfasst. Jedenfalls fehlt es aber an der vergleichbaren Wertungslage. ${ }^{61}$ Die Durchführung der Reise inklusive der Beförderung fällt in den Risikobereich des Reiseveranstalters,

57 Für die Beschädigung des Hotels ebenso: Tonner (Fn. 16), $\$ 651$ h Rn. 45.

58 Steinrötter (Fn. 8), \$651 h Rn. 46.

59 Im Ergebnis ebenso: Löw, Pauschalreiserecht (Fn. 53), NJW 2020, 1252 (1253); Staudinger/Achilles-Pujol (Fn. 10), $\$ 7$ Rn. 27.

60 Grundlegend: C.-W. Canaris, Die Feststellung von Lücken im Gesetz, 2. Aufl. Berlin 1983.

61 Dazu näher: P. Meier/F. Jocham, Rechtsfortbildung - Methodischer Balanceakt zwischen Gewaltenteilung und materieller Gerechtigkeit, JuS 2016, 392 (396f.). 
der bei Fehlern nach $\$ 651$ i BGB auf Grund der Mangelrechte hierfür einzustehen hat. Es ist deshalb angezeigt, dem Reiseveranstalter den Lohn vollends zu versagen, wenn der Kunde wegen solcher Umstände, die ansonsten einen Mangel begründen würden, den Rücktritt schon vor Beginn der Reise erklärt. ${ }^{62} \mathrm{Maßnahmen,} \mathrm{die} \mathrm{nach} \mathrm{der} \mathrm{Rückkehr} \mathrm{des} \mathrm{Reisenden} \mathrm{an}$ seinen Heimatort eintreten, fallen dagegen in dessen Risikobereich. Ob der Reisende später einer Quarantäne unterliegt, steht außerhalb des Einflussbereichs des Reiseveranstalters. Er muss hierfür auch nicht objektiv einstehen, weil er lediglich die Erbringung der Reise als solche versprochen hat, nicht aber auch dafür garantiert, dass der Reisenden im späteren Verlauf keine Nachteile befürchten muss. Die Reise ist mit der Rückbeförderung abgeschlossen, weshalb auch die Einstandspflicht des Reiseveranstalters in diesem Moment endet. Es ist daher geboten, Aspekte, die die Durchführung der Reise selbst nicht betreffen, im Rahmen des Rücktritts vor Reisebeginn unberücksichtigt zu lassen und somit dem Reiseveranstalter einen Anspruch auf angemessene Entschädigung nach $\$ 651 \mathrm{~h}$ Abs. 1 S. 3 BGB zu gewähren. Die Ausnahmevorschrift des $\$ 651$ h Abs. 3 BGB, die als solche zwar nicht generell analogieunfähig, ${ }^{63}$ aber im Allgemeinen eng zu verstehen ist, ${ }^{64}$ darf auf diese Konstellation nicht erstreckt werden. Drohen dem Reisenden erst Einschränkungen nach Abschluss der Pauschalreise, verbleibt es bei den allgemeinen Regeln, so dass eine Kündigung des Reisenden eine Entschädigungspflicht zu Gunsten des Reiseveranstalters auslöst.

62 So im Ergebnis auch Harke (Fn. 8), $\$ 651$ h Rn. 45, der davon ausgeht, eine Erheblichkeit bestehe erst, wenn der Reisende den Reisepreis um wenigstens $50 \%$ mindern könne.

63 Meier/Jocham, Rechtsfortbildung (Fn. 61), JuS 2016, 392 (395); F. Rosenkranz, Die Auslegung von „Ausnahmevorschriften“, JURA 2015, 783 (787); D. Schneider, Singularia non sunt extendenda, JA 2008, 174 ff.; M. Würdinger, Ausnahmevorschriften sind analogiefähig!, JuS 2008, 949.

64 Dazu M. Würdinger, Die Analogiefähigkeit von Normen. Eine methodologische Untersuchung über Ausnahmevorschriften und deklaratorische Normen, AcP 206 (2006), 946 (960f.). 


\section{Insolvenzsicherung}

\section{Geltende Lage im deutschen Recht}

Sollte es im Zuge der Corona-Krise zu einer Insolvenz eines Reiseveranstalters kommen, rückt die Vorschrift des $\$ 651 \mathrm{r}$ BGB in den Blickpunkt. Sie verpflichtet in ihrem Abs. 1 den Reiseveranstalter, für eine Insolvenzabsicherung zu sorgen, die dafür einsteht, dass der Reisende insoweit den Reisepreis erstattet bekommt, als Reiseleistungen infolge der Insolvenz entfallen sind ${ }^{65}$ oder der Reisende nochmals an einzelne Leistungserbringer Zahlungen entrichten muss. ${ }^{66}$ Zugelassen als Insolvenzabsicherer sind nach $₫ 651$ r Abs. 2 S. 1 BGB ausschließlich in Deutschland zum Betrieb befugte Versicherungen oder Kreditinstitute. Dies scheint auf den ersten Blick den Reisenden umfassend zu schützen. Als problematisch und für den Kunden potentiell riskant erweist sich allerdings die Beschränkung des $\$ 651$ r Abs. 3 S. 3 BGB, wonach der Kundengeldabsicherer seine Einstandspflicht auf 110 Millionen $€$ je Jahr begrenzen darf. Bereits die Insolvenz des Reiseveranstalters Thomas Cook hat belegt, dass diese Grenze offensichtlich weit unter den drohenden Schäden im Falle einer Insolvenz angesetzt ist. ${ }^{67}$ Betrachtet man exemplarisch die TUI $A G$, zeigt sich, dass diese im Geschäftsjahr 2018/2019 einen Umsatz von über 18 Milliarden $€$ erwirtschaftete, ${ }^{68}$ so dass das Schadenspotenzial durch die Versicherungshöchstgrenze noch nicht einmal annähernd abgedeckt ist. Bei der Schaffung der Grenze des $₫ 651$ r Abs. 3 S. 3 BGB hat der Gesetzgeber schlicht die unter altem Recht bestehende Summe fortgeschrieben und sich dabei an den bisherigen Schadensereignissen orientiert. ${ }^{69}$

65 Dazu näher: D. Blankenburg, in: B. Gsell/W. Krüger/S. Lorenz/C. Reymann (Hrsg.), beckonline.Grosskommentar zum Zivilrecht, 2020, $\$ 651$ r BGB Rn. 30 ff.

66 Siehe hierzu: Blankenburg (Fn. 65), \$651 r BGB Rn. $32 \mathrm{ff}$.

67 Siehe dazu auch: BT-Drs. 19/16990, 3.

68 So der Geschäftsbericht der TUI AG.

69 BT-Drs. 18/10822, 89. 


\section{Europarechtliche Vorgaben}

Die Europarechtskonformität der deutschen Umsetzung wurde und wird allerdings wiederholt ${ }^{70}$ und zu Recht bestritten. ${ }^{71}$ Art. 17 Abs. 2 S. 1 Pauschalreise-RL gebietet, dass die Insolvenzsicherheit wirksam sein und zudem in der Lage sein muss, die nach vernünftigem Ermessen voraussehbaren Kosten abzudecken. Nach dem erläuternden Erwägungsgrund 40 der Pauschalreise-RL ist beabsichtigt, den Schutz derart zu bemessen, dass alle vorhersehbaren Zahlungen inklusive der Kosten der Rückbeförderung abgedeckt werden. Dazu soll der Gesamtumsatz des jeweiligen Reiseveranstalters in den Blick genommen werden, um anhand dessen bestimmen zu können, welche Summe für die Insolvenzabsicherung erforderlich ist. Nur sehr unwahrscheinliche Risiken dürfen außer Betracht bleiben. Hierunter fallen nach Erwägungsgrund 40 insbesondere die gleichzeitige Insolvenz mehrerer Reiseveranstalter. Betrachtet man im Verhältnis zu den europäischen Vorgaben jedoch die deutsche Vorschrift, so zeigt sich, dass eine Grenze, die lediglich geringfügig mehr als ein halbes Prozent des Jahresumsatzes des größten Reiseveranstalters abdeckt, keinesfalls genügen kann, um den Anforderungen der Pauschalreise-RL gerecht zu werden. ${ }^{72}$ Spätestens seit der Insolvenz des Reiseveranstalters Thomas Cook im September 2019 wurde die Ineffizienz des deutschen Rechts an dieser Stelle offenkundig. ${ }^{73}$ Gleichwohl hat der Gesetzgeber nicht reagiert und insoweit keine Vorkehrungen getroffen, so dass zumindest im Anschluss an dieses Ereignis eine Begrenzung auf 110 Millionen $€$ nicht nur ganz unwahrscheinliche Risiken außer Acht lässt. Vor den Anforderungen des Art. 17 Abs. 2

70 P. Hueck, Neues Pauschalreiserecht, alte Insolvenzabsicherungsproblematik - Vorschläge für einen unionsrechtskonformen Reisendenschutz in Deutschland, RRa 2019, 254 (255 ff.); B. M. Quarch, Rezension zu Reiserecht - Handbuch des Pauschalreise-, Reisevermittlungs-, Reiseversicherungs- und Individualreiserechts, NZV 2020, 34; Staudinger (Fn. 16), \16 Rn. 23; A. Staudinger, Kundengeldabsicherung bei Pauschalreisen sowie Luftbeförderung de lege lata und ferenda, $r+s$ 2018, 2 (5); K. Tonner, Die Pauschalreiserichtlinie lässt dem Umsetzungsgesetzgeber kaum Spielraum - Zum RegE eines Dritten Reiserechtsänderungsgesetzes, RRa 2017, 5 (8); Tonner (Fn. 16), $\$ 651$ r Rn. 24; offen: A. Baumgärtner, in: H. G. Bamberger/H. Roth/W. Hau/R. Poseck (Hrsg.), Beck'scher Online-Kommentar BGB, $\$ 651$ r Rn. 41.1.

71 Differenzierend: Blankenburg (Fn. 65), $\$ 651 \mathrm{r}$ Rn.67, der vor der Insolvenz von Thomas Cook die Europarechtskonformität bejahte, nun aber ausschließt.

72 Zweifelnd auch: Baumgärtner (Fn. 70), \$651 r Rn. 41.1.

73 Ebenso: Baumgärtner (Fn. 70), $\$ 651 \mathrm{r}$ Rn.41.1; Blankenburg (Fn. 65), $\$ 651 \mathrm{r}$ Rn. 67. 
S. 1 Pauschalreise-RL kann demnach $₫ 651$ r Abs. 3 S. 3 BGB keinen Bestand haben. ${ }^{74}$

\section{Rechtsfolgen}

\section{Verpflichtung des Absicherers}

Als problematisch erweisen sich jedoch die Rechtsfolgen des Verstoßes gegen die RL. Die Beschränkungsmöglichkeit in $\$ 651 \mathrm{r}$ Abs. 3 S. 3 BGB bezieht sich zunächst auf die Verpflichtung des Reiseveranstalters. ${ }^{75}$ Es stellt sich in diesem Zusammenhang die Frage, ob Kunden, wenn der Insolvenzabsicherer seine Haftung durch Vereinbarung mit dem Reiseveranstalter auf die genannte Summe begrenzt hat, dennoch von diesem in voller Höhe die Begleichung ihrer Ansprüche verlangen können. ${ }^{76}$ Nach $\$ 651 \mathrm{r}$ Abs. 4 S. 1 BGB muss der Reisevermittler dafür sorgen, dass der Reisende einen unmittelbaren Anspruch gegen den Insolvenzabsicherer erlangt. ${ }^{77}$ Es ergeben sich damit direkte Forderungsrechte zwischen Kunde und Sicherungsgeber. Zudem kann sich wegen $\$ 651$ r Abs. 4 S. 2 BGB der Insolvenzabsicherer nicht auf Einreden berufen, die aus dem Vertrag zwischen ihm und dem Reiseveranstalter herrühren. Dieses allgemeine Verbot wird allein über die von $\$ 651$ r Abs. 3 S. 3 BGB zugelassene Möglichkeit der Begrenzung eingeschränkt, so dass auch diese mit der Existenz der Norm steht und fällt. In der Rechtsprechung des EuGH ist anerkannt, dass derartige beschränkende Normen, soweit sie sich als europarechtswidrig erweisen, unangewendet bleiben müssen und damit die europäischen Vorgaben mittelbar auch belastend für Private wirken können. ${ }^{78}$ Dem soll das Verbot, Pflichten von Privaten direkt aus einer unmittelbar anzuwendenden

74 Wie hier: Hueck, Insolvenzabsicherungsproblematik (Fn. 70), RRa 2019, 254 (255 ff.); Quarch, Rezension (Fn. 70), NZV 2020, 34; Staudinger (Fn. 16), $\$ 16$ Rn. 23; ders., Kundengeldabsicherung (Fn. 70), r+s 2018, 2 (5); Tonner, Spielraum (Fn. 70), RRa 2017, 5 (8); ders. (Fn. 16), \$651 r Rn. 24.

75 Nur der Reiseveranstalter ist nach $\$ 651$ r Abs. 1 S. 1 BGB dem Kunden gegenüber verpflichtet.

76 In der Insolvenz von Thomas Cook hat sich die Frage nicht gestellt, da die Bundesregierung angekündigt hat, alle Schäden selbst zu übernehmen. Siehe dazu die Mitteilung der Bundesregierung vom 11.12.2019.

77 Insoweit wird ein Vertrag zu Gunsten Dritter nach $₫ 328$ BGB abgeschlossen. Vgl. hierzu: Baumgärtner (Fn. 70), \$ 651 r Rn. 29; Steinrötter (Fn. 8), \651 r Rn. 25; Tonner (Fn. 16), \$ 651 r Rn. 26.

78 EuGH Slg. 2010, I-393 Rn. 44 ff. 
Richtlinie abzuleiten, ${ }^{79}$ nicht entgegenstehen, ${ }^{80}$ wenn die grundlegende Obligation bereits im nationalen Recht angelegt ist. ${ }^{81}$ So liegt der Fall hier, weshalb der Kundengeldabsicherer unbegrenzt gegenüber den Reisenden haften muss. Die vermeintliche Beschränkung wirkt daher wegen $₫ 651 \mathrm{r}$ Abs. 4 S. 2 BGB nicht zu seinen Gunsten, mit der Folge, dass der Sicherungsgeber für alle Schäden einzustehen hat, die bei einem Reisenden anfallen. ${ }^{82}$ Weil der nationale Gesetzgeber eine feste Grenze gezogen und damit keine ordnungsgemäße Wertentscheidung getroffen hat, kann die Einschränkung auch nicht im Rahmen dessen, was von Art. 17 Abs. 2 S. 1 Pauschalreise-RL noch als zulässig angesehen wird, aufrechterhalten bleiben. ${ }^{83}$ Insoweit wäre nämlich entweder eine auf jedes Unternehmen spezifisch zugeschnittene Höchstgrenze oder eine prozentuale Beschränkung bezogen auf den Umsatz des Reiseveranstalters erforderlich. Weil aber der Gesetzgeber in $\$ 651 \mathrm{r}$ BGB keine dieser Maßnahmen ergriffen hat und die starre Grenze auf Grund europäischen Rechts unangewendet bleiben muss, existiert kein Anknüpfungspunkt für eine geltungserhaltende Reduktion. Es steht nämlich nicht fest, was der deutsche Gesetzgeber entschieden hätte, wenn er eine europarechtskonforme Regelung getroffen hätte. Jede erhaltende Maßnahme würde sich daher als Spekulation darstellen und sich damit vom Willen des Gesetzgebers lösen. Hierfür besteht allerdings weder Anlass noch Befugnis, so dass $₫ 651 \mathrm{r}$ Abs. 3 S. 3 BGB im Wege der europarechtskonformen Rechtsfortbildung ${ }^{84}$ in Gänze unangewendet bleiben muss. Eine Einstandspflicht des Versicherers in voller Höhe ist damit unvermeidlich.

79 EuGH, Slg. 1986, 723 Rn. 48; Slg. 1994, I-3325 Rn. 20 ff.; Slg. 2005, I-3565 Rn. 73.

80 M. Nettesheim, in: E. Grabitz/M. Hilf/M. Nettesheim, Das Recht der Europäischen Union, Bd. I, 68. EL: 2019, Art. 288 AEUV, Rn. 163; M. Ruffert, in: C. Calliess/M. Ruffert, EUV/AEUV, 5. Aufl. 2016, Art. 288 AEUV, Rn. 81; dazu aber krit.: $M$. Hilf, Die Richtlinie der EG - ohne Richtung, ohne Linie?, EuR 1993, 1 (10 f.).

81 Insoweit besteht nach dem EuGH eine Verpflichtung zur Fortbildung des nationalen Rechts innerhalb der Grenzen des methodisch Erlaubten: EuGH Slg. 1999, I-905 Rn. 22 ff.; Slg. 2000, I-4941 Rn. 30; Slg 2004 I-8835 Rn. 113 ff.; Slg. I 2006, $6091 \mathrm{Rn} .108 \mathrm{ff}$.

82 In diese Richtung auch: Baumgärtner (Fn. 70), $\$ 651 \mathrm{r}$ Rn. 41.1; letztlich ebenso durch eine europarechtskonforme Rechtsfortbildung: Staudinger/Achilles-Pujol (Fn. 10), $\$ 7$ Rn. 68.

83 Wohl ebenso: Baumgärtner (Fn. 70), $\$ 651$ r Rn. 41.1.

84 Siehe dazu allgemein: C. Herresthal, Rechtsfortbildung im europarechtlichen Bezugsrahmen, München 2006. 


\section{Staatshaftung}

\section{a) Gegenüber den Reisenden}

Für die Bundesrepublik Deutschland besteht darüber hinaus in doppelter Hinsicht die Gefahr einer potentiellen Staatshaftung. Kann der Kundengeldabsicherer, der nur auf eine Höchstsumme von 110 Millionen $€$ eingestellt war, nicht sämtliche Ansprüche der Reisenden erfüllen, steht diesen ein unmittelbarer Regressanspruch gegen den Staat zu. Nach der Rechtsprechung des EuGH ergibt sich insoweit direkt aus Europarecht eine Verpflichtung zum Ausgleich für legislatives Unrecht, wenn Mitgliedstaaten eine Richtlinie nicht korrekt umsetzen. ${ }^{85}$ Ein ausreichend qualifizierter Verstoß ist zu bejahen, da spätestens seit der Insolvenz von Thomas Cook der bereits seit Langem von einer Vielzahl von Autoren beklagte ${ }^{86}$ Missstand offenkundig ist, ohne dass der Gesetzgeber Maßnahmen ergriffen hätte. ${ }^{87}$ Zudem ist die mangelnde Geeignetheit der deutschen Maßnahmen schon bei einem nur oberflächlichen Vergleich zwischen Umsatz und damit potentiellem Insovenzschaden und Absicherungshöhe erkennbar. Der zu ersetzende Schaden besteht darin, dass bei zutreffender Umsetzung der Absicherer gezwungen gewesen wäre, umfangreichere Rücklagen zu bilden und etwaige Rückversicherungen abzuschließen, so dass er auch alle an ihn gestellten Ansprüche erfüllen könnte. Kommt es hierzu auf Grund des staatlichen Versagens nicht, hat die Bundesrepublik Deutschland den Differenzbetrag als Schaden zu erstatten.

\section{b) Gegenüber dem Absicherer}

Zugleich können aber auch Forderungen des Sicherungsgebers an die Bundesrepublik Deutschland gestellt werden. ${ }^{88}$ Dieser wird nämlich in seinem Vertrauen auf die korrekte Umsetzung enttäuscht, wenn er sich auf

85 Siehe bereits zu bisherigen Fällen der Staatshaftung bei fehlender Insolvenzsicherung im Reiserecht: EuGH Slg. 1996 I-4845; Slg. 1999, I-3499; BeckRS 2014, 80245; NJW-RR 2019, 940 (941 f.). Ebenfalls eine Staatshaftung der Bundesrepublik für diesen Fall bejahend: Baumgärtner (Fn. 70), $\$ 651$ r Rn. 41.1; zweifelnd: Blankenburg (Fn. 65), $\$ 651$ r Rn. 69.1 f.; Staudinger (Fn. 16), $\$ 12$ Rn. 23.

86 Siehe zur früheren Diskussion: Tonner (Fn. 16), $\$ 651$ r Rn. 21.

87 Dies sieht nun auch Blankenburg (Fn. 65), $\$ 651 \mathrm{r}$ Rn. 69.2 als problematisch an. Wie hier auch: Staudinger/Achilles-Pujol (Fn. 10), \$ 7 Rn. 70

Ebenso: Baumgärtner, $\$ 651$ r (Fn. 70), Rn. 41.1. 
die durch $\$ 651 \mathrm{r}$ Abs.3 S.3 BGB ausgesprochene Haftungsobergrenze nicht verlassen kann, sondern in deutlich größerem Umfang als nach seiner begründeten Kalkulation vorgesehen, herangezogen wird. In den Entscheidungen des EuGH ist bislang nur die Situation behandelt worden, dass einem Privaten Vorzüge aus einer Richtlinie deshalb entgehen, weil sie nicht in das nationale Recht implementiert wurden. ${ }^{89}$ Gleichwohl muss aber ein Schadensersatzanspruch nach denselben Regeln auch dann gewährt werden, wenn sich eine Person auf die durch das nationale Gesetz ausgesprochenen Regelungen verlässt und insoweit Dispositionen triff, die sich später wegen der Europarechtswidrigkeit der nationalen Norm als verfehlt erweisen..$^{90}$ Der Bürger ist nicht weniger schutzwürdig, wenn er einen durch das europäische Recht vorgesehenen Anspruch nicht erlangt, als wenn er auf vermeintlich wirksame Zusagen, die durch das nationale Recht abgegeben werden, vertraut. ${ }^{11}$ Auch in diesem Fall muss ihm ein finanzieller Ausgleich gewährt werden, weil seine Nachteile auf der fehlerhaften Umsetzung des europäischen Rechts beruhen. Der Schaden des Absicherers besteht zumindest darin, dass er andernfalls weitaus höhere Prämien hätte durchsetzen können. Die Differenz zwischen den verlangten und den bei voller Absicherung angemessenen Prämien hat ihm die Bundesrepublik Deutschland zu ersetzen. Kann der Kundengeldabsicherer nachweisen, dass er bei Kenntnis der vollen Einstandspflicht weitergehende Maßnahmen, insbesondere Absicherungen über Rückversicherungen, getroffen hätte, muss ihm der Staat auch die hieraus entstehenden Schäden kompensieren. Diese belaufen sich auf den durch die Rückversicherung erstatteten Beträge abzüglich der im Vorfeld gezahlten Prämien.

\section{Vertragsverletzungsverfahren}

Schließlich droht auch ein Vertragsverletzungsverfahren gem. Art. 258 AEUV durch die EU-Kommission, so dass dem nationalen Gesetzgeber dringend anzuraten ist, $\mathbb{} 651$ r Abs. 3 S. 3 BGB schnellstmöglich derart an-

89 Grundlegend: EuGH, Slg. 1991, I-5357.

90 T. Giegerich, Europarechtskonträre Staatshaftung? - Widerspruchsfreiheit der Rechtsordnung und Pflichtenstellung Privater nach dem Karlsruher HoneywellDictum, EuR 2012, 373 ff.; P. Meier, Der Widerruf eines Teilzeit-Wohnrechte-Vertrags nach der Verbraucherrechterichtlinie, ZfIR 2014, 799 (803).

91 Giegerich, Staatshaftung (Fn. 90), EuR 2012, 373 ff.; Meier, Widerruf (Fn. 90), ZfIR 2014, 799 (803); zustimmend auch Baumgärtner (Fn. 70), \$ 651 r Rn. 41.1. 
zupassen, dass er die Vorgaben des Art. 17 Pauschalreise-RL korrekt in das nationale Recht transformiert.

\section{E. Anspruch auf Rückzahlung des Reisepreises}

\section{Aktuelle Lage}

Kann die Reise insgesamt oder in Teilen auf Grund der gesundheitlichen Lage oder behördlicher Verbote nicht durchgeführt werden, liegt ein Reisemangel vor. Ein solcher besteht nämlich auch dann, wenn eine Reise überhaupt nicht erbracht wird oder sogar unmöglich ist, so dass es auf deren Antritt nicht ankommt. Diese zum alten Recht noch durchaus umstrittene Frage ${ }^{92}$ ist nunmehr legislativ in $₫ 651$ i Abs. 2 S. 3 BGB entschieden. Es sind demnach die gesetzlichen Mangelrechte in dieser Situationen heranzuziehen; ${ }^{93}$ das allgemeine Leistungsstörungsrecht findet keine Anwendung. ${ }^{94}$ Der Reisende kann mithin die Rückzahlung nicht schon nach $\$ 326$ Abs. 4 i.V.m. $\$ 346$ Abs. 1 BGB verlangen, sondern ist darauf verwiesen, den Vertrag nach $₫ 651$ i Abs. 3 Nr. 5 i.V.m. $\$ 6511$ Abs. 1 S. 1 BGB zu kündigen, um so die Vertragsbeziehung insgesamt zum Erlöschen zu bringen. Geleistete Zahlungen sind, soweit die Reise insgesamt nicht erbracht werden kann, ${ }^{95}$ komplett nach $\$ 6511$ Abs. 2 S. 2 HS. 2 BGB zu erstatten. Alternativ kann der Kunde nach $\$ 651 \mathrm{~m}$ Abs. 2 i.V.m. $₫ 346$ Abs. 1 BGB die Rückgewähr des bisher gezahlten Preises wegen der nach $\$ 651 \mathrm{~m}$ Abs. 1 S. 1 BGB eintretenden Minderung verlangen. Fällt die Reise insgesamt aus, beträgt der Minderungsbetrag stets die volle Höhe. ${ }^{96}$ Weil die Minderung ipso iure wirkt, ${ }^{97}$ muss der Reisende keine weiteren Maßnahmen ergreifen, um einen Anspruch auf Rückzahlung zu erlangen. Er steht somit im Ergebnis ebenso wie bei der Anwendung von $\$ 326$ Abs. 1 S. 1

92 Dazu A. Teichmann, Die Struktur der Leistungsstörungen im Reisevertrag, JZ 1979, $737 \mathrm{ff}$.

93 So bereits unter altem Recht: BGHZ 97, 255 (259 ff.); BGHZ 100, 157 (180 f.).

94 Geib (Fn. 6), $\$ 651$ i Rn. 3; Sprau (Fn. 49), \$651 i Rn. 2; Tonner (Fn. 16), \$651 i Rn. 34.

95 Soweit Teile schon erbracht sind, bleibt der Anspruch nach $₫ 651$ Abs. 2 S. 1 BGB bestehen.

96 Ebenso: A. S. Kramer, in: B. Gsell/W. Krüger/S. Lorenz/C. Reymann (Hrsg.), beckonline.Grosskommentar zum Zivilrecht, 2020, $\$ 651 \mathrm{~m}$ BGB Rn. 127 (für eine Teilleistung).

97 Staudinger (Fn. 20), $\$ 651$ m Rn. 1, Steinrötter (Fn. 8), \651 m Rn. 8. 
BGB. Die Rückgewähr ist grundsätzlich in Geld geschuldet. ${ }^{98}$ Andere Angebote des Reiseveranstalters, insbesondere Gutscheine, muss der Reisende nicht akzeptieren..$^{99}$ Es steht ihm allerdings natürlich gleichwohl frei, eine alternative Leistung an Erfüllung statt nach $\$ 364$ BGB anzunehmen; ${ }^{100}$ der Reiseveranstalter kann ihn hierzu aber nicht zwingen.

Grundsätzlich dasselbe gilt, wenn lediglich die isolierte Beförderung geschuldet ist. Gemäß Art. 5 Abs. 1 lit. a i.V.m. Art. 8 Abs. 1 lit. a der Fluggastrechteverordnung hat der Reisende bei der Annullierung des Fluges Anspruch auf die volle Erstattung des für das Ticket gezahlten Preises. Dasselbe gilt nach Art. 19 Abs. 4 VO 181/2011 für Busreisen, wobei dessen Abs. 5 S. 4 sogar explizit bestimmt, dass die Rückzahlung in Geld zu erfolgen hat. Art. 18 Abs. 2 VO 1177/2010 regelt die Rückgewähr des Reisepreises für den Fall einer Nichtbeförderung mit einem Binnenschiff, wobei Art. 18 Abs. 3 VO 1177/2010 dem Beförderer als Erstattungsmodalitäten ausschließlich die Barzahlung, die elektronische Überweisung, die Gutschrift oder die Leistung per Scheck erlaubt und so ebenfalls deutlich macht, dass der Reisende nur Geld akzeptieren muss. Allein bei einer Reise mit der Bahn ergibt sich der Erstattungsanspruch nicht klar aus europäischem Recht. Art. 32 CIV, auf den Art. 15 VO 1371/2007 verweist, enthält nur Regelungen zum Schadensersatz, nicht aber auch solche für die Rückzahlung des Fahrpreises bei einem vollständigen Entfall der Leistung. Auch aus Art. 16 lit. a VO 1371/2007 wird man eine derartige Verpflichtung nicht entnehmen können. ${ }^{101}$ Die Norm richtet sich bereits nach ihrem Wortlaut ausschließlich auf eine Verspätung, nicht aber auch auf die Annullierung; ${ }^{102}$ gegen ihre analoge Anwendung spricht, dass die Annullierung durch das CIV bestimmt wird. Zwar sind dort keine Regeln zur Rückzahlung des Fahrpreises getroffen, so dass die internationalen Vorgaben insoweit keine Sperrwirkung für das europäische Recht entfalten können. Allerdings hat sich der europäische Gesetzgeber einer Regelung der Annullierung insgesamt enthalten und in diesem Bereich allein auf die

98 Geib (Fn. 6), $\$ 651$ m Rn. 11; Kramer (Fn. 96), \$ 651 m Rn. 176; Steinrötter (Fn. 8), $\$ 651 \mathrm{~m} \mathrm{Rn.} \mathrm{30;} \mathrm{Staudinger} \mathrm{(Fn.} \mathrm{16),} \$ 21$ Rn. 32.

99 Kramer (Fn. 96), $\$ 651$ m BGB Rn. 176; Steinrötter (Fn. 8), $\$ 651$ m Rn. 30; Tonner (Fn. 16), $\$ 651 \mathrm{~m} \mathrm{Rn.} 19$.

100 Dazu: Staudinger/Achilles-Pujol (Fn. 10), $\$ 7$ Rn. 19 ff.

101 Unklar aber die Bekanntmachung der Kommission vom 18.03.2020 „Interpretative Guidelines on EU passenger rights regulations in the context oft he developing situation with Covid-19“, Nr. 4.2, die nicht eindeutig macht, ob sie Art. 16 auch auf Annullierungen anwenden will.

102 Lindemann, Fahrgastrechte (Fn. 27), TranspR 2011, 10 (13); Meier (Fn. 27), $\$ 18$ b Rn. 44. 
Normen des CIV verwiesen. Es ist deshalb systematisch nicht angezeigt, eine Vorschrift über die Verspätung entsprechend anzuwenden. Vielmehr verhält sich die Verordnung selbst zur Annullierung überhaupt nicht, weshalb aus ihr auch keine Vorgaben für derartige Konstellationen abgeleitet werden können. Auf Grund der Lücke, die sowohl das internationale Übereinkommen als auch die europarechtliche Verordnung lassen, verbleibt es beim nationalen Recht. ${ }^{103}$ Weil der Vertrag über den Transport von Menschen werkvertraglichen Charakter hat ${ }^{104}$ und damit die $\$ \$ \$ 631 \mathrm{ff}$. BGB anzuwenden sind, kann der Unternehmer die Zahlung erst verlangen, wenn er selbst die von ihm geschuldete Leistung erbracht hat. Da diese jedoch insgesamt nicht durchgeführt werden kann, wird sie nach $\$ 275$ Abs. 1 BGB unmöglich, so dass auch der Anspruch auf die Gegenleistung nach $\$ 326$ Abs. 1 S. 1 BGB entfällt. Dies gilt hier auch vor dem Hintergrund, dass Beförderungsleistungen von der h. M. nicht als absolute Fixgeschäfte eingeordnet werden. ${ }^{105}$ Wird nämlich der Flug nicht verschoben, sondern entfällt er komplett, liegt eine Annullierung vor, die dazu führt, dass dieser Flug nicht mehr ausgeführt werden kann und damit dauerhaft unterbleibt. ${ }^{106}$ Infolgedessen tritt hinsichtlich der gebuchten Beförderung Unmöglichkeit ein. Der Rückzahlungsanspruch ergibt sich demnach im deutschen Recht aus $\$ 326$ Abs. 4 i.V.m. $\$ 346$ Abs. 1 BGB.

\section{Reformüberlegungen des Gesetzgebers}

\section{Rechtspolitische Planungen}

Um die Reiseveranstalter zu schützen und um ihre Liquidität zu sichern, erwägt der Bundesgesetzgeber allerdings eine Änderung des BGB, wonach

103 Vgl. zur Beziehung zwischen nationalem und internationalem Recht im Bereich der Bahnreisen: Meier (Fn. 27), $\mathbb{1 8}$ b Rn. 7; im Ergebnis wie hier: A. Staudinger, Zweifelsfragen der Verordnung (EG) Nr.1371/2007 des Europäischen Parlaments und des Rates vom 23. 10. 2007 über die Rechte und Pflichten der Fahrgäste im Eisenbahnverkehr, EuZW 2008, 751 (753).

104 BGHZ 62, 71 (75); BGH NJW 1969, 2014 (2015).

105 BGH NJW 2009, 2743 (2743 f.); OLG Frankfurt NJW 2018, 3591; ausführlich dazu: A. Staudinger, Wider die Qualifikation des Luftbeförderungsvertrages als absolutes Fixgeschäft sowie die Relevanz des Mängelrechts, RRa 2005, 249 (251 ff.); anders noch: OLG Düsseldorf NJW-RR 1997, 930; OLG Frankfurt a. M. NJWRR 1997, 1136.

106 Siehe zur Unterscheidung zwischen Annullierung und Verspätung: EuGH, Slg. 2009, I-10923 Rn. 36. 
bei Reiseausfällen auf Grund der Corona-Krise der Reiseveranstalter statt der Rückgewähr in Geld Gutscheine an den Reisenden überlassen kann. ${ }^{107}$ Auch wenn die genauen Details bislang offen sind, ${ }^{108}$ stellt ein solches Vorgehen in jedem Fall eine spürbare Beschränkung der Rechte der Reisenden dar. Der Gutschein hat darlehensähnlichen Charakter, ${ }^{109}$ weil der Reisende die Geldzahlung bereits jetzt erbringt, die Gegenleistung in Form der Reiseleistung aber erst in mehr oder weniger ferner Zukunft zu erhalten hoff. Insoweit trägt der Reisende das Insolvenzrisiko des Reiseveranstalters, der möglicherweise in der Zwischenzeit seinen Geschäftsbetrieb einstellen muss und daher seine Verpflichtung nicht mehr erfüllen kann. Zwar dürften auch die Gutscheine von der Insolvenzsicherung des $\$ 651 \mathrm{r}$ BGB erfasst sein, ${ }^{110}$ allerdings geht jedenfalls von der Begrenzung des $₫ 651$ r Abs. 3 S. 3 BGB eine erhebliche Gefahr für den Reisenden aus. Dies gilt selbst, wenn sie europarechtskonform gestaltet wäre, ${ }^{111}$ weil auch dann ein vom Richtliniensetzer toleriertes Restrisiko verbleibt. Darüber hinaus hat der Reisende möglicherweise Zins- und Inflationsnachteile. Er kann die Summen nicht anderweitig investieren und erleidet unter Umständen einen Nachteil schon deshalb, weil bis zur Einlösung des Gutscheins eine Geldentwertung erfolgt ist. Nicht zuletzt widerspricht die Gutscheinlösung in einigen Fällen auch insoweit den Interessen des Reisenden, weil er nur einmalig für diese Reise mit diesem Reiseveranstalter buchen wollte oder auf absehbare Zeit keinen weiteren Urlaub mehr plant. Dies dürfte vor allem bei Reisen aus besonderen Anlässen (z.B. Hochzeitsreise) der Fall sein, die in aller Regel nicht zu einem anderen Zeitpunkt nachgeholt werden; ob der Reisende eine andere Reise in vergleichbarem Umfang plant oder wünscht, ist offen und kann möglicher-

107 Siehe dazu die Mitteilung bei becklink 2016104.

108 Siehe den Beschluss des „Corona-Kabinetts“ vom 02.04.2020 und die Pressemitteilung des Presse- und Informationsamts der Bundesregierung Nr. 118.

109 So auch die Stellungnahme der Verbraucherzentralen, becklink 2015987.

110 Weil die Norm auf den „Reisepreis“ Bezug nimmt, dürfte es wohl keine Rolle spielen, ob dieser schon mit einer bestimmten, konkretisierten Reiseleistung in Verbindung steht oder vorerst abstrakt für eine noch näher auszugestaltende Verpflichtung geleistet wird. Eine solche Auslegung gebietet jedenfalls der Sinn und Zweck der Norm, die den Reisenden schützen will, weil er regelmäßig weit im Vorfeld leisten muss (Baumgärtner (Fn. 70), \$651 r Rn. 1; Steinrötter (Fn. 8), $\$ 651$ r Rn. 4). Unklar aber Staudinger (Fn. 16), $\$ 12$ Rn. 12, der davon ausgeht, nur solche Leistungen seien erfasst, die nach dem Abschluss des Pauschalreisevertrages erbracht werden. Siehe nunmehr aber: Staudinger/Achilles-Pujol (Fn. 10), $\$ 7$ Rn. $61 \mathrm{ff}$.

111 Dazu oben: D. II. und III. 
weise aktuell auch noch nicht bestimmt werden. Wie sich die Lage des Reisenden in Zukunft darstellen und ob er über die Möglichkeiten verfügt, eine vergleichbare Reise erneut anzutreten, ${ }^{112}$ wird auf diese Weise seinem Risiko überantwortet. Auch wenn der Gesetzgeber durch staatliche Garantien oder besondere Möglichkeiten, dennoch die Auszahlung zu fordern, einen Teil der Nachteile abwenden würde, ${ }^{113}$ verbliebe letztlich gleichwohl eine spürbare Verschlechterung der Position des Reisenden.

\section{Rechtliche Bewertung}

Die rechtspolitischen Fragestellungen können aber im Ergebnis offenbleiben. Eine derartige Änderung kann durch den deutschen Gesetzgeber unabhängig von verfassungsrechtlichen Fragen ${ }^{114}$ ganz überwiegend selbstständig nicht erfolgen, weil sie sich in Widerspruch zum europäischen Recht setzen würde. ${ }^{115}$ Dies wird besonders plastisch für die Flug-, Busund Binnenschifffahrtsreisen. Hier ergeben sich die Rechte des Kunden unmittelbar aus einer europäischen Verordnung, die durch den deutschen Gesetzgeber bereits kompetenziell nicht geändert werden kann. ${ }^{116}$ Eingriffe des Bundestages verbieten sich an dieser Stelle von vornherein.

Doch auch im Reisevertragsrecht lassen sich die angedachten Änderungen nicht durch eine Entscheidung des nationalen Gesetzgebers verwirklichen. Die Verpflichtung zur Rückzahlung in Geld ist nämlich durch die Pauschalreise-RL determiniert und entzieht sich daher einer Gestaltung durch die deutsche Legislative. Gem. Art. 4 Pauschalreise-RL ist diese vollharmonisierend, so dass dem nationalen Gesetzgeber weder Abweichun-

112 Dabei kommt es nicht zuletzt auch auf die Frage an, ob der oder die Reisenden später noch Zeit finden werden, eine Reise dieses Umfangs anzutreten.

113 In diese Richtung weisen zumindest die Beschlüsse des „Corona-Kabinetts“ vom 02.04.2020 und die Pressemitteilung des Presse- und Informationsamts der Bundesregierung Nr. 118.

114 Diese bestehen vor allem in den Fragen zur Zulässigkeit der Rückwirkung. Siehe dazu: H. Maurer, Staatsrecht I, 6. Aufl. München 2010, $\$ 17$ Rn. 101 ff.; H. Sodan/ J. Ziekow, Grundkurs öffentliches Recht, 8. Aufl. München 2018, \$ 7 Rn. 45 ff.

115 Anders aber unter Bezugnahme auf Wertungen des Primärrechts: Staudinger/ Achilles-Pujol (Fn. 10), \$7 Rn. $114 \mathrm{ff}$.

116 Siehe auch die Bekanntmachung der Kommission vom 18.03.2020 „Interpretative Guidelines on EU passenger rights regulations in the context oft he developing situation with Covid-19“, Nr. 2.2. 
gen in die eine noch in die andere Richtung gestattet sind. ${ }^{117}$ Zudem ist der völlige Ausfall der Reise vom Anwendungsbereich der RL erfasst, wie Art. 13 Abs. 3 Pauschalreise-RL klarstellt, der allgemein von Vertragswidrigkeiten spricht und damit deutlich macht, dass der von $\$ 651$ i Abs. 2 S. 3 BGB gewählte Mangelbegriff gleichermaßen das europäische Recht beherrscht. Es darf demnach auch bei einer generellen Unmöglichkeit der Reise vom Pflichtenprogramm der RL nicht abgewichen werden. Art. 13 Abs. 6 UAbs. 1 Pauschalreise-RL sieht die Befugnis des Reisenden zum Rücktritt vor, wenn die Mangelhaftigkeit erheblich ist und nicht in zureichender Zeit Abhilfe geschaffen wird. Dabei dürfte das europäische Recht so zu verstehen sein, dass eine Fristsetzung gleichwohl entbehrlich ist, wenn die Abhilfe unmöglich ist, auch wenn dies im Wortlaut nicht zum Ausdruck kommt. ${ }^{118}$ Unabhängig davon spielt dieser Aspekt aber für die Frage, ob dem Reisenden die Rückzahlung in Geld geschuldet ist, keine Rolle. Weil explizit der Rücktritt vorgesehen ist, muss der Reisende das zurückerhalten, was er zuvor an den Reiseveranstalter überlassen hat. Art. 12 Abs. 2 S. 2 Pauschalreise-RL spricht explizit aus, dass der Reisende die Erstattung aller Leistungen verlangen kann, so dass hier erkennbar ein Recht zur Erbringung von Gutscheinen nicht vorgesehen ist. Mithin kommt die Gewährung eines Gutscheins höchstens dann in Betracht, wenn der Reisende zuvor einen solchen eingesetzt und damit den Reisepreis beglichen hat, ${ }^{119}$ weil dann auch keine ursprüngliche Zahlung durch ihn vorliegt, die gem. Art. 12 Abs. 2 S. 2 Pauschalreise-RL zwingend zu erstatten wäre. In allen anderen Fällen hat der Reisende Geld geleistet und muss deshalb auch solches wiedererhalten. ${ }^{120}$ Dasselbe gilt für die in Art. 14 Abs. 1 Pauschalreise-RL europarechtlich vorgesehene Minderung. Die Regelung sieht vor, dass für die Dauer der Vertragswidrigkeit der Reisende eine angemessene Preisminderung erhält. Fällt die Reise gänzlich aus, kann nur die vollständige Minderung angemessen sein. Hat der Reisende aber das Recht,

117 Dazu näher: E. Führich, Die neue Pauschalreiserichtlinie, NJW 2016, 1204 (1205); K. Tonner, Der Vorschlag einer neuen Pauschalreiserichtlinie, ZRP 2014, $5(6)$.

118 Im Ergebnis ebenso für den Bereich des $\$ 6511$ BGB: Deppenkemper (Fn. 6), $\$ 6511$ Rn. 7; Geib (Fn. 6), $\$ 6511$ Rn. 7; V. Klingberg, in: B. Gsell/W. Krüger/S. Lorenz/C. Reymann (Hrsg.), beckonline.Grosskommentar zum Zivilrecht, 2020, \6511 BGB Rn.43; Staudinger (Fn. 16), \20 Rn. 18; Steinrötter (Fn. 8), $\$ 6511$ Rn. 21.

119 Hierzu: AG Hannover, RRa 2002, 79 (80); allgemein: M. Zwickel, Vertragsbeziehungen, Leistungsstörungen und Gestaltungsmöglichkeiten beim Gutscheingeschäft, NJW 2011, 2753 (2757).

120 Ebenso: Staudinger/Achilles-Pujol (Fn. 10), $\$ 7$ Rn. 18. 
die Zahlung gänzlich zu unterlassen, sind bereits erbrachte Leistungen ohne Rechtsgrund gewährt worden. Korrespondierend, wenn auch nicht explizit in der RL ausgesprochen, muss er deshalb einen Anspruch auf Rückzahlung dieser Summen haben. ${ }^{121}$ Insoweit kann eine angemessene Minderung ebenfalls nur in einer Rückzahlung in Geld bestehen, weil der Reisende ansonsten keine äquivalente und damit angemessene Preisreduktion erhält, wenn er Gutscheine akzeptieren muss. Solche sind aus den oben genannten Gründen nicht gleichwertig zur Barzahlung, weshalb sich keine Angemessenheit im Verhältnis zum kompletten Unterbleiben der Reise ergäbe.

Eine Regelung im BGB, wonach der Reisende Gutscheine statt der Barleistung vom Reiseveranstalter annehmen muss, verstößt mithin gegen die zwingenden Vorgaben der Pauschalreise-RL. Dies gilt auch dann, wenn eine staatliche Absicherung vorliegt, weil diese dennoch nicht gleichwertig mit der Zahlung von Geld ist und zudem eine solche Option durch die RL nicht vorgesehen wird. Eine Anpassung wäre nur dann zulässig, wenn die Richtlinie durch die Europäische Union geändert würde. Bis dahin würde sich ein nationaler Alleingang als Verletzung des Sekundärrechts darstellen und damit einer Prüfung durch den EuGH nicht standhalten. Hieran kann auch das europäische Primärrecht nichts ändern. ${ }^{122}$ Die Ausgestaltung der Pauschalreise-RL ist nicht für sich genommen unverhältnismäßig, da die Rückzahlung eines bereits im Vorfeld entgegengenommenen Preises in diesen Fällen dem Wesen der werkvertraglichen Leistung des Unternehmers entspricht. Kann er seine Leistung nicht erbringen, verliert er auch den Anspruch auf die Gegenleistung, wobei es dann nicht dem Verhältnismäßigkeitsgrundsatz widerspricht, wenn er infolge seines unternehmerischen Risikos auch die Gefahr eingeht, insolvent zu werden. Dies ist jeder werkvertraglichen Betätigung immanent und Bestanteil der vereinbarten Risikotragung.

Ein Spielraum besteht für den deutschen Gesetzgeber lediglich im Bereich der Eisenbahnbeförderung. Dort existieren, wie gezeigt, keine europäischen Vorgaben über die Rückzahlungsverpflichtung, so dass diese allein auf nationalem Recht beruht. Der deutsche Gesetzgeber wäre daher darin frei, eine entsprechende Sonderregelung zu treffen. Diese wäre auch nach überwiegender Meinung mit Art. 3 Abs. 1 GG zu vereinbaren, weil

121 Im Ergebnis ebenso zum deutschen Recht: Geib (Fn. 6), \$651 m Rn. 11; Kramer (Fn. 96), $\$ 651 \mathrm{~m} \mathrm{Rn.} \mathrm{176;} \mathrm{Steinrötter} \mathrm{(Fn.} \mathrm{8),} \$ 651 \mathrm{~m} \mathrm{Rn.} \mathrm{30;} \mathrm{Staudinger} \mathrm{(Fn.} \mathrm{16),}$ $\$ 21 \mathrm{Rn} .32$.

122 So aber Staudinger/Achilles-Pujol (Fn. 10), \ 7 Rn. $114 \mathrm{ff}$. 
zwar tatsächlich eine Ungleichbehandlung zwischen den Bahn- und anderen Transportunternehmen bestünde, ${ }^{123}$ diese allerdings auf den Entscheidungen unterschiedlicher Legislativorgane beruhen würde, und eine solche Ungleichbehandlung von vornherein durch Art. 3 Abs. 1 GG nicht verboten sein soll. ${ }^{124}$

Als Konsequenz dieser Schwierigkeiten wird derzeit der Plan durch die Bundesregierung auch nicht mehr verfolgt.

\section{Konsequenzen einer möglichen Änderung}

Für die Bürger würde sich, falls sich der nationale Gesetzgeber zu einer Änderung entschließen sollte, dennoch wohl eine erhebliche Verschlechterung ergeben. Weil der Gesetzgeber in Kenntnis der Vorgaben des Europarechts das nationale Recht umgestalten würde, wäre eine europarechtskonforme Rechtsfortbildung ausgeschlossen. Diese ist auch nach der Rechtsprechung des EuGH nur zulässig, soweit die nationale Methodenlehre sie erlaubt. ${ }^{125}$ Nachdem sich allerdings die Rechtsfortbildung nicht in Widerspruch zum legislativen Willen setzen darf, ${ }^{126}$ der grundsätzlich nicht „objektiv“ als vermeintlicher Wille des Gesetzes, ${ }^{127}$ sondern „subjektiv“ als Wille des Gesetzgebers bestimmt werden muss, ${ }^{128}$ wäre hier eine derartige

123 Insoweit lassen sie sich unter den gemeinsamen Begriff der „Beförderer“ fassen. Siehe zur Struktur der Oberbegriffsbildung: W. Heun, in: H. Dreier, Grundgesetz-Kommentar, 3. Aufl. Tübingen 2013, Art. 3 Rn. 24.

124 So BVerfGE 110, 412 (439); krit. dazu aber bspw.: C. Riese/P. Noll: Europarechtliche und verfassungsrechtliche Aspekte der Inländerdiskriminierung, NVwZ 2007, 516 (520 f.); F. Wollenschläger, in: P. M. Huber/A. Voßkuhle (Hrsg.), von Mangoldt/Klein/Starck, Grundgesetz, Bd. 1, 7. Aufl. München 2018, Art. 3 Rn. 221.

125 EuGH Slg 2005, I-5285 Rn. 47; Slg. 2006, I-6057 Rn. 110; so auch zum deutschen Recht BVerfGK 19, 89 (100).

126 Siehe zur grundsätzliche Unzulässigkeit der Rechtsfortbildung contra legem: Meier/Jocham, Rechtsfortbildung (Fn. 61), JuS 2016, 392 (394).

127 Hierfür aber bspw.: F. Bydlinski, Juristische Methodenlehre und Rechtsbegriff, 2. Aufl. Nachdruck, Wien, New York 2011, S. 453 ff.; G. Hirsch, Auf dem Weg zum Richterstaat? Vom Verhältnis des Richters zum Gesetzgeber in unserer Zeit, JZ 2007, 853 (855); W.-R. Schenke, Verfassung und Zeit, AöR 103 (1978), 566 (580 f.).

128 BVerfGE 128, 193 (210 f.); 132, 99 (127); 134, 204 (238); BVerfG NZS 2015, 502 (503); NJW 2015, 1359 (1367). 
Fortbildung unzulässig, ${ }^{129}$ da sich der Gesetzgeber ganz explizit in Widerspruch zu den europäischen Regelungen setzen will. ${ }^{130}$ Dies schließt eine Veränderung des nationalen Rechts durch eine Rechtsfortbildung auch nach europäischem Recht aus. Zudem kann ein Anspruch gegen die Reiseveranstalter nicht direkt aus der Richtlinie folgen, weil nach der Rechtsprechung des EuGH unmittelbare Pflichten zwischen Privaten nicht aus einer Richtlinie abgeleitet werden dürfen. ${ }^{131}$ Dem Bürger bliebe demnach nur noch der vom EuGH entwickelte Staatshaftungsanspruch gegen die Bundesrepublik Deutschland, ${ }^{132}$ um den vorsätzlichen Verstoß gegen das europäische Recht zumindest finanziell auszugleichen. Der notwendige qualifizierte Verstoß stünde außer Zweifel, weil dem Gesetzgeber die Rechtswidrigkeit seines Handelns sogar positiv bekannt wäre, so dass kein Anlass besteht, ihn von der Haftung für diesen zu befreien. Hiernach müsste die Bundesrepublik sämtliche Forderungen der Reisenden in Geld ersetzen und könnte im Gegenzug lediglich die Übereignung der ausgekehrten Gutscheine verlangen. ${ }^{133}$ Darüber hinaus wäre ein Vertragsverletzungsverfahren durch die EU-Kommission vor dem EuGH gegen die Bundesrepublik Deutschland denkbar (Art. 258 AEUV).

\section{F. Schluss}

Das Reiserecht ist in der Corona-Krise besonders gefordert. Die hier aufgegriffenen Beispiele erschöpfen die Fragen, die sich aktuell und in Zukunft stellen, keineswegs und sollen lediglich einen Einblick in die Breite des Gebiets geben. Angesichts der erheblichen Summen und der Vielzahl an betroffenen Verträgen wird auf die Gerichte in den kommenden Monaten und Jahren eine Flut an Verfahren zukommen, die zum Teil auch neue Rechtsfragen aufwerfen werden. Diese müssen in Kooperation zwischen Wissenschaft und Rechtspraxis einer adäquaten Lösung zugeführt werden.

129 So auch C. Herresthal, Voraussetzungen und Grenzen der gemeinschaftsrechtskonformen Rechtsfortbildung, EuZW 2007, 396 (400); ders., Die richtlinienkonforme und die verfassungskonforme Auslegung im Privatrecht, JuS 2014, 289 (292f.).

130 Siehe dazu auch: BGH, BKR 2020, 253 (254f.).

131 EuGH, Slg. 1986, 723 Rn. 48; Slg. 1994, I-3325 Rn. 20 ff.; Slg. 2005, I-3565 Rn. 73.

132 Siehe bereits zu bisherigen Fällen der Staatshaftung bei fehlender Insolvenzsicherung im Reiserecht: EuGH Slg. 1996 I-4845; Slg. 1999, I-3499; BeckRS 2014, 80245; NJW-RR 2019, 940 (941 f.).

133 So auch zur parallelen Problematik der fehlerhaften Anlageberatung: BGHZ 79, 337 (346); 123, 106 (110); BGH NJW 2006, 2042 (2043). 